\title{
On the unification of nuclear-structure theory: $A$ response to Bortignon and Broglia
}

\author{
Norman D. Cook ${ }^{\mathrm{a}}$ \\ Department of Informatics, Kansai University, Osaka, Japan
}

Received: 25 July 2016

Published online: 9 September 2016

(C) The Author(s) 2016. This article is published with open access at Springerlink cor Communicated by T. Biro

\begin{abstract}
Nuclear-structure theory is unusual among the diverse fields of quantu. hysics. Although it provides a coherent description of all known isotopes on the basis of a uan mechanical understanding of nucleon states, nevertheless, in the absence of a fundamental theory $c_{t}$ ear force acting between nucleons, the prediction of all ground-state and excited-state nuclear bi ng energies is inherently semiempirical. I suggest that progress can be made by returning to found conal work of Eugene Wigner from 1937, where the mathematical symmetries of nucleon states rery defined. Those symmetries were later successfully exploited in the development of the independent-1 artiele model (IPM $\sim$ shell model), but the geometrical implications noted by Wigner were negl-at. Here review how the quantum-mechanical, but remarkably easy-to-understand geometrical in rpres on of the IPM provides constraints on the parametrization of the nuclear force. The proposed c metr al IPM" indicates a way forward toward the unification of nuclear-structure theory that Bo- ignon oroglia have called for.
\end{abstract}

\section{Introduction}

The "challenge" posed by Bortignon ara Broglia for achieving unification of specifically nu lear-structure theory with nuclear-reaction data shoul be applauded by all physicists. Their suggestion for "nu theorists to take center stage" is an unusual, welcome invitation to return to the basics of nuclear prys, while using the conceptual and computation tool developed in the 21st century to solve old an vet man wed problems. I would therefore like to tal the portunity of their call for "a new type of nucl theoret ran who computes less and thinks more" to por out the early thinking of Eugene Wigner, ans what it 1 , plies for computational nuclearstructure or tollay.

After mo than seven decades of semi-quantitative "modeli ,", nu ear-structure physics is arguably unique amo tural sciences in lacking a unifying theory within hich rigorous computational models can be coherently organized. Atomic physics, chemistry, solid-state physics, and even molecular biology, each have unifying theories within which most experimental and computational efforts are now made, but nuclear-structure theory remains a collection of (literally) dozens of mutually contradictory models [2]. The core problem since the $1930 \mathrm{~s}$ has been the absence of a quantitative understanding of the nuclear force itself.

\footnotetext{
a e-mail: cook@res.kutc.kansai-u.ac.jp
}

While many researchers await developments in quark theory for elucidation of the character of the nucleonnucleon interaction, it is worth bearing in mind that nuclear-structure theorists from the heyday of nuclear theory (1950-1970) have repeatedly stated their conviction that insights from high-energy particle physics are unlikely to shed light on the relatively low-energy phenomena of nuclear structure. As noted by Bortignon and Broglia [1], the so-called "unified" model of Bohr and Mottelson [3] from the 1960s was a success in providing a means to address both the collective and the independent-particle aspects of nuclei, but the nucleon-clustering phenomena treated in the alpha-cluster and boson models remained outside of the "unified" model and progress in clarifying the nuclear force did not follow from their work. The related problem of the mean-free-path of nucleons in stable nuclei (long, as in a gas? or short, as in a liquid?) has also remained unresolved. Further unification is yet possible [1].

Today, few nuclear physicists would espouse the need for additional models, but it is a historical fact that, while liquid- and gaseous-phase models have been given abundant consideration, the profoundly simple solid-phase (lattice) model of Wigner [4] has remained largely overlooked. In a foundational work on nuclear symmetries, published in Physical Review in 1937 [4], Wigner outlined a geometrical interpretation of the quantal symmetries of the independent-particle model (IPM). As a theorist, Wigner 


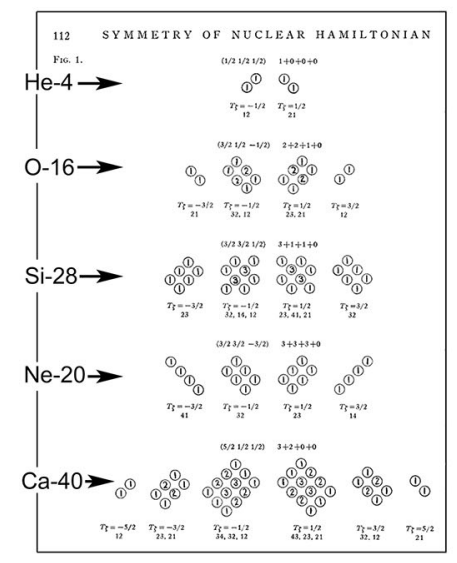

(A) Wigner (1937)

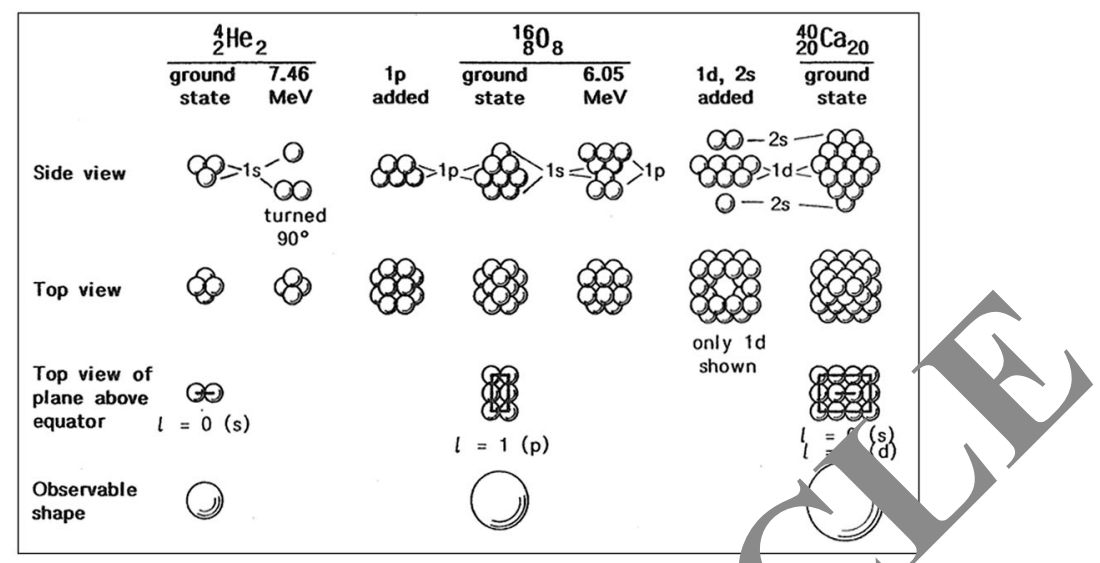

(B) Everling (1958-2008)

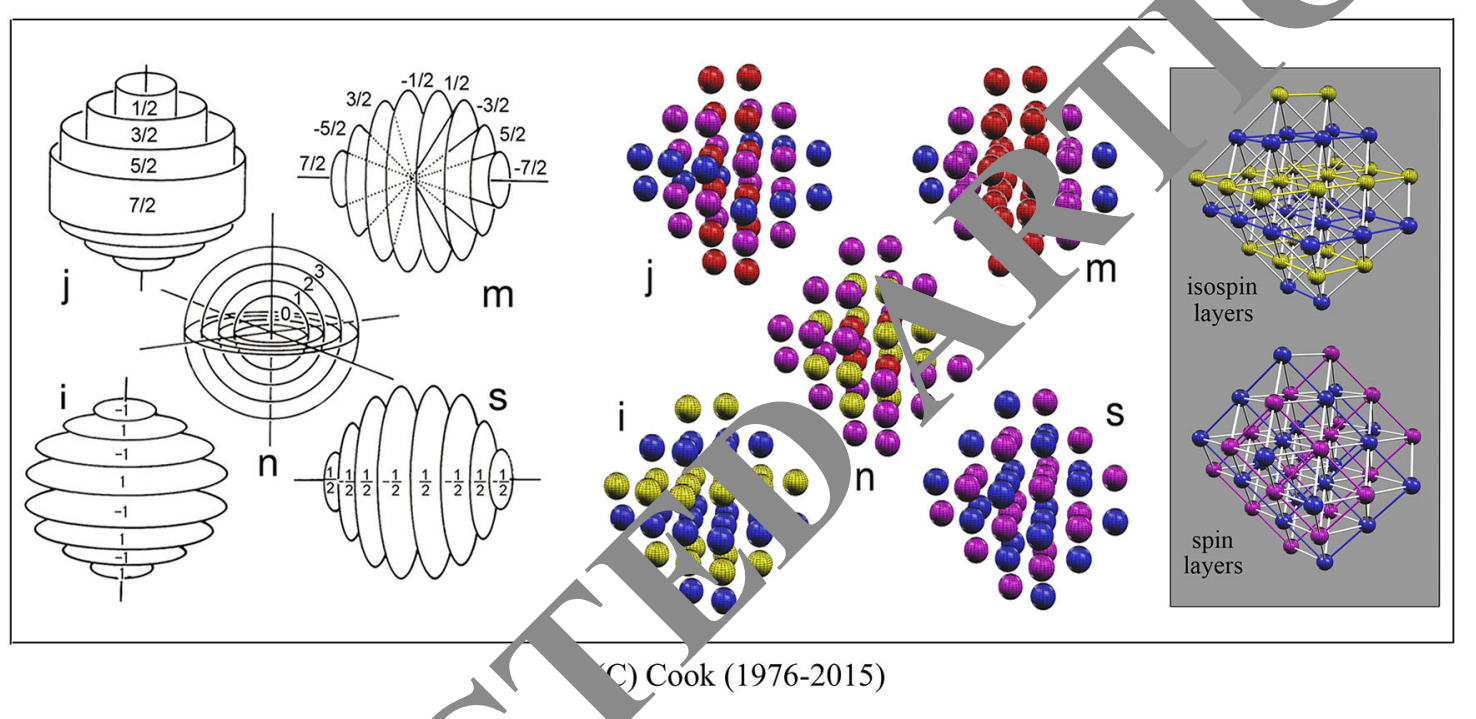

Fig. 1. The fcc lattice symmetries of nu eon quanturn states. (A) In a 1937 article in Physical Review entitled Symmetry of the nuclear Hamiltonian, Wigner depicte the nudleon eigenvalues of the first three, doubly magic $n$-shells, noting that they form a face-centered close-packed lattice [ $s_{\mathrm{S}} \mathrm{D}$ ) wverling [5] subsequently showed the geometry of the $s-, p$ - and $d$-subshells of the same nuclei. (C) Using compute aphics techniques, Cook [7-9] illustrated the quantum value symmetries of the first four $n$-shells.

was clearly interested or whe abstract symmetries of the quantum nu ibers, be did in fact note that the quantal regulari ies f nuclesn states have a remarkable, inherent 3D shucture at is identical to a face-centeredcubic (fcc) attice with orthogonal spin and isospin layering (fig. 1 . In evitably, the first impression of such visual victi the nucleus is one of classical mechanics out vigney s main argument concerned the quantal sym rus wherent to the nuclear Hamiltonian. Whatever ma be the correct physical interpretation of those symmetries, they are fundamentally a consequence of the quantum texture (subscripts $n, l, j, m, s, i$ and parity) that is the essence of the Schrödinger wave equation used in nuclear quantum mechanics.

It is in fact uncertain what Wigner himself thought with regard to the physical significance of the lattice representation of nuclear structure, but the identity between the fcc lattice and the well-established IPM has been independently pointed out several times since then: Everling in
1958 [5], Lezuo in 1974 [6], Cook in 1976 [7-9], Dallacasa in 1981 [10], and many others sporadically since then. All five of the above authors have emphasized the geometrical simplicity of the empirically known symmetries of the nucleon quantum numbers that are a fundamental aspect of conventional nuclear-structure theory. They have shown that all of the nucleon quantum numbers have unambiguous geometrical definitions within the framework of a lattice model of (low-energy) nuclear structure (eqs. (1)-(7)).

More than a decade after Wigner's innovative explication of nuclear symmetries, the idea of spin-orbit coupling was established by Mayer and Jensen, and the labeling of nucleon quantal variables was changed to accommodate the shell model. Wigner's pre-eminent contribution to a quantum-mechanical understanding of nuclear structure was, however, recognized by the Nobel Committee in awarding half of the 1963 Physics Prize to Wigner and a quarter each to Mayer and Jensen. Since then, details of the isomorphism between one particular lattice structure 
(the antiferromagnetic fcc lattice with spin and isospin layering) and the known symmetries of the nucleus have been published many times (e.g., [4-10], with a full list of references through 2010 in [7-9]).

Unfortunately, Wigner himself did not elaborate on the underlying geometry of the IPM - and it has fallen to others to note that conventional nuclear theory can be recast from the abstract higher dimensionality of the symmetries of quantum mechanics to comprehensible (if not classical) three-dimensional geometry. The seeming complexity of any 3D lattice of nucleons belies a remarkable simplicity, insofar as all of the shells and subshells of the wellestablished IPM have geometrical interpretations that reflect the symmetries that are in daily use by all IPM practitioners. These symmetries are easily summarized, as in eqs. (1)-(7):

$$
\begin{aligned}
& \text { principal, } n=(|x|+|y|+|z|-3) / 2, \\
& \text { orbital angular momentum, } l=(|x|+|y|) / 2, \\
& \text { total angular momentum, } j=(|x|+|y|-1) / 2, \\
& \text { azimuthal, } m=|x| *(-1)^{(x-1) / 2} / 2, \\
& \text { spin, } s=(-1)^{(x-1) / 2} / 2, \\
& \text { isospin, } i=(-1)^{(z-1) / 2}, \\
& \text { parity, } \pi=\operatorname{sign}(x * y * z),
\end{aligned}
$$

where all quantum numbers are defined in terms of eacb nucleon's unique set of $x, y, z$ coordinates in Cartesian space. All of the nucleon shells/subshells and their sccupancies are thereby reproduced (eqs. (1)-(4)). Tha indicates that the IPM and the lattice model are fun. mentally isomorphic, but they clearly differ mplyin a diffuse, gaseous nuclear interior, on tho one $\mathrm{nd}$, or a high-density nuclear interior where $\mathrm{p}$ acleon-nucle on interactions are local, on the other. U: ng a "mean-field" approximation, the gaseous-phase IPM s bech the dominant model since the $1950 \mathrm{~s}$, but has not veen reconciled with the known nuclear force, know. from nucleon-nucleon scattming ex periments (fig. 2).

In contrast, the latt e h s a lis aid-drop-like texture that is consistent with dimensions of the nuclear force. The pri nary rea nfor not exploring the solidphase model is t $\mathrm{t}^{\mathrm{C}}$ orrect assumption that the nuclear "shell" struct re dem do the gas-like orbiting of nucleons. The g ometrical brald-up of nuclei in the fcc lattice, however, $\mathrm{Cl}_{-}$de onstrates that such an assumption is unfou $d$.

Te h ve in act frequently published (e.g., [5-10]) on the is orpmsm between the IPM and the fcc lattice - to the coln ive yawn of the community of nuclear-structure theorists. Although it is understandable that theorists are not enthusiastic about "unconventional" approaches to the problems of nuclear structure, it is worth reiterating that the original idea was proposed by Eugene Wigner -whose "conventional" work forms the basic quantummechanical understanding of the nucleus! It is Wigner's remarkable insight that there is a fundamental geometry to the texture of the nuclear interior. The significance of that geometry is, to be sure, not yet fully understood,

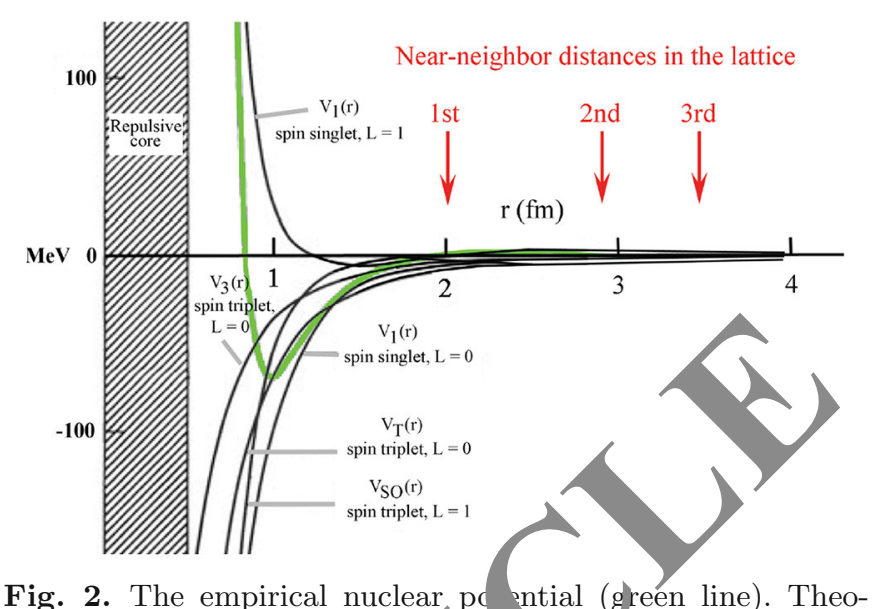

Fig. 2. The empirical nuclear po ntial (green line). Theoretical studies on the nuclear rce tulse strongly attractive and repulsive compone ts a to quark interactions (black lines). At the mean dist ces betw $y$ nucleons, as implied by both the liquid-drop roa nd the lattice model, a few $\mathrm{MeV}$ per "bond" suffices to achie waclear binding.

but one notable vlication of the lattice geometry of the nucleus c cerns th, nuclear force.

The grpss racteristics of the nuclear force are well known and have been reproduced in various theoretical ols (Bor A, Argonne, Paris, Idaho, etc.) over the course of th past 40 years (fig. 2). Alternative parametrizaons re of course possible, depending on the spin/isospin co ponents of the nuclear force that are specified, but bf particular interest for both the liquid-drop conception of nuclear structure and lattice models is the fact that the nucleon-nucleon interaction at the distances of 1st, 2nd or 3rd nearest neighbors in a close-packed lattice of nucleons is weak $\left(\left|E_{\mathrm{BE}}\right|<5 \mathrm{MeV}\right)$. Such small values for nucleon-nucleon interactions are fully consistent with what is known about nuclear binding energies and excited states, but are orders of magnitude smaller than theoretical quark effects.

While some theorists may be reluctant to "return" to Wigner's ideas from 1937 [4], it is relevant to note that, while subsequent experimental progress in measuring the nuclear force has been remarkable, comparable theoretical progress has not been achieved. On the contrary, textbooks on nuclear physics typically note that, because of the inherent complexity of the nuclear many-body problem, theorists have found it necessary to rely on admittedly imperfect nuclear models specifically because fundamental questions concerning the nuclear force remain unanswered. As a consequence, theoretical work on the 2-body nucleon interaction itself has been slow since the 1950s, and the theoretical focus has become mean-field approximations and the conceptually obscure, computationally difficult topics of 3- and 4-body forces.

Contrary to first impressions, recasting nuclear-structure theory within the lattice representation of quantal symmetries is a less radical renovation of nuclear-structure theory than is currently appreciated — principally because the lattice symmetries map directly onto the known quantal symmetries of the nucleus. The properties of the nu- 
clear force known from nucleon-nucleon scattering experiments fit neatly with either the liquid-drop or the lattice perspective on nuclear structure, but are explicitly rejected by gaseous-phase models that rely on the physically unrealistic mean-field theory to study nucleon interactions.

In conclusion, while research on the quark constituents of hadrons in high-energy physics has had some success in classifying particles in the Standard Model, it appears that the low-energy realm of nuclear-structure physics requires the attention of "a new type of nuclear theoretician" who "thinks more" about the self-consistency of nuclearstructure theory [1].

Open Access This is an open access article distributed under the terms of the Creative Commons Attribution License (http://creativecommons.org/licenses/by/4.0), which permits unrestricted use, distribution, and reproduction in any medium, provided the original work is properly cited.

\section{References}

1. P.F. Bortignon, R.A. Broglia, Eur. Phys. J. A 52, 64 (2016).

2. W. Greiner, J.A. Maruhn, Nuclear Models (Springer, New York, 1996).

3. A. Bohr, B.R. Mottelson, Nuclear Structure (Benjamin, New York, 1975).

4. E. Wigner, Phys. Rev. 51, 106 (1937).

5. F. Everling, notarized document (1958) an na v rêferences at http://www.everling-nuclear-latt -mocel. com/ (2016).

6. K.J. Lezuo, Atomkernenergie 23, 285 974).

7. N.D. Cook, Atomkernenergie 28 195 (1)

8. N.D. Cook, V. Dallacasa, Phys Rev. C 361883 (1987).

9. N.D. Cook, V. Dallacasa $\Lambda$ dels of the Atomic Nucleus, 2nd edition (Spring Ne arl, 2010) available at www.res.kutc.kansai/as p/ cook/PDFs/man2.pdf.

10. V. Dallacasa, Atom' nenergic 1, 143 (1981).

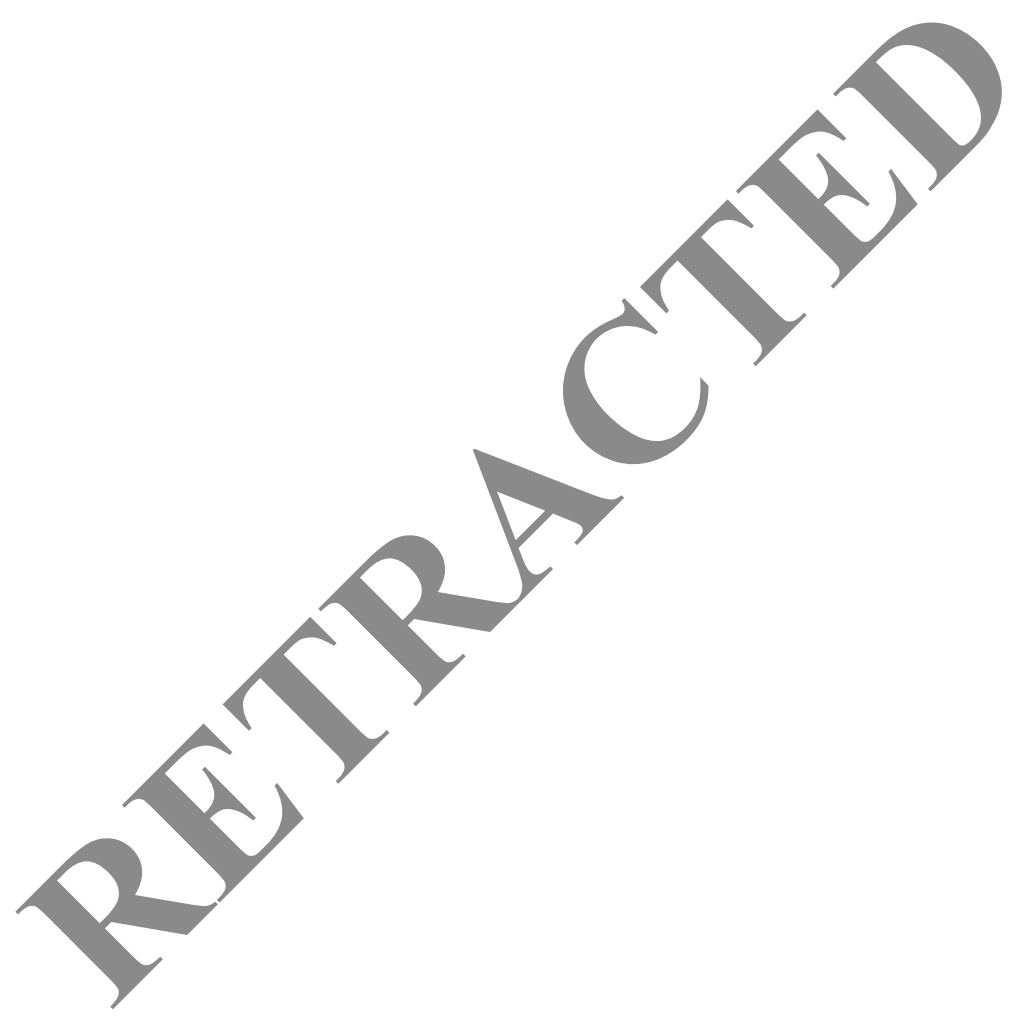

